\title{
Study on Proofreading and Error Correction in TEM-8 From Error Analysis
}

\author{
Hui Liu ${ }^{1}$ and Yingliang Liu ${ }^{1, *}$ \\ ${ }^{1}$ School of Foreign Languages, Wuhan University of Technology, Wuhan, Hubei 430000, China \\ *Email: yliu@whut.edu.cn
}

\begin{abstract}
Proofreading and error correction in TEM-8 papers is a challenging part for Chinese learners majoring in English. Accordingly, this study explores targeted language forms encompassed in proofreading and error correction of TEM-8 papers from the year 2012 to 2019. The paper then analyzes errors in proofreading and error correction from the perspective of Error Analysis to minimize negative transfer from L1 to English L2 learners. The results show that the proofreading and error correction tests examinees' comprehensive language ability from lexical, syntactic and textual aspects. The outcome of this study is inspirational to English majors so that they can analyze their own errors in language structures, to progress in proofreading and error correction consciously.

Keywords: Proofreading and Error Correction, Error Analysis, Transfer, Richards' Error Type
\end{abstract}

\section{INTRODUCTION}

Proofreading and error correction is a question type in TEM-8 papers, which is closely related to reading and writing skills of language learners. Not only does it involve mental and cognitive language processing, but also it calls for a profound and thorough capacity of comprehending main ideas, appreciating authors' attitudes, proficiently applying connecting devices such as cohesion and recursiveness, and appropriately utilizing grammatical rules [1]. The examination syllabus of TEM-8 was changed in 2016. Accordingly, proofreading and error correction was renamed language use after the year of 2016. To make it specific, the former term "proofreading and error correction" is used in this paper.

On the one hand, proofreading and error correction is an indispensable part of TEM-8 papers, and it has always been a challenging part for English majors. On the other hand, English learners can make a profit by a conscious identification of targeted language forms in proofreading and error correction, as well as an analysis of these errors employing Richards' error type.

Research concerned about the impact of Error Analysis on language learning and teaching reports a positive effect. However, few studies analyze classification of language forms included in proofreading and error correction of TEM-8 from Error Analysis. This paper intends to make up for the deficiency, aiming to help English majors who are going to take the TEM-8 test in 2021 raise their awareness about strategies of tackling proofreading and error correction more efficiently.

\section{LITERATURE REVIEW}

\subsection{Error Analysis}

Error Analysis (EA), proposed by S. Pit Corder, is the first approach to the study of SLA which includes an internal focus on learners' creative ability to construct language [2]. Based on the description and analysis of actual learner errors in L2, Error Analysis considers the process of second language acquisition as one of acquiring rules. That means learners consistently make hypotheses about rules in target languages through input, and then testify and modify them [3]. What is widely accepted is that errors can be divided into intralingual errors, meaning resulting from within the language itself, as opposed to interlingual errors coming from differences between L1 and L2. As for why errors occur during the process of acquiring a second language, different linguists have various opinions. James (1998) considers the ultimate cause of these errors L2 learners make as the ignorance of a certain language item in target languages, which is the preliminary explanation of errors for his part [4]. Richards (1971) shows a more wide-ranging consideration of error types in his paper $A$ non-contrastive approach to error analysis [5]. From his new perspective, errors are composed of four types: overgeneralization, ignorance of rule restriction, incomplete application of rules and false concepts hypothesized.

In the past few decades, there has been tons of research on Error Analysis at home, some of which deal with error classification. For example, based on Universal Grammar of Chomsky, Peng Jing classifies errors into several kinds from a cognitive perspective and adds some error types, 
claiming that UG and EA play a significant part in second language acquisition and language teaching and learning, despite the fact that UG and EA have been controversial [6]. More studies focus on the enlightenment of EA to English teaching for L2 learners in particular. Zhang Haiyan applies Error Analysis Theory to English writing teaching for postgraduates of non-English majors through analyzing the source, type and cause of errors, concluding that L2 learners are able to avoid mistakes resulting from overgeneralization, ignorance of rule restrictions, incomplete application of rules, and false concepts hypothesized [7].

\subsection{Previous Research on Proofreading and Error Correction}

Proofreading and error correction is such a comprehensive question type as testing test-takers' reading and writing skills. The part of proofreading and error correction enjoys a profound and stable seat in TEM- 8 even though the test pattern of TEM- 8 has been modified several times, thanks to the validity and reliability of testing students' English proficiency of language learning.

Not only in TEM-8, but also in many large-scale English examinations such as National Matriculation English Test (NMET) and College English Test band 6 (CET-6), proofreading and error correction is widely and repeatedly used. Therefore, studies on proofreading and error analysis are abundant, which are conducted from various angles. For these three kinds of examinations, the vast majority of research addresses strategies and methods of how to answer the proofreading and error analysis questions. Similarly, numerous studies deal with the classification of errors in proofreading and error correction and attribution of errors test-takers make. In addition, some studies center on the validity and reliability of proofreading and error correction in various kinds of examinations, a few of which discuss whether or not proofreading and error correction should be included in the entrance examination for college. Still, analysis of proofreading and error correction from textual cohesion is also conducted.

From above, it is concluded that research on classification of errors, strategies and methods of how to answer the proofreading and error analysis question amounts to more than 70 percent. Later, with the development of linguistics, we can witness an increasing number of studies on validity and research employing corpus-based approach.

\section{CLASSIFICATION OF ERRORS}

This study cites the proofreading and error correction in the TEM-8 papers from 2012 to 2019 , adding up to eight examination papers. Language forms in proofreading and error correction which were quoted are categorized closely, some findings of which are as follows.

The errors that test-takers are supposed to figure out and correct in proofreading and error correction can be classified into three sorts roughly: lexical errors, syntactic errors and textual errors.

According to Table1, it can be found that lexical errors amount to half of all errors in proofreading and error correction. And they are mostly further divided into such smaller categories as mistaken use of words, redundancy, absence and mistaken use of parts of speech, which are listed from a larger quantity to a smaller one. Among these sub-categories, the mistaken use of words, including verbs, nouns and prepositions, occupies this section by a majority of twenty-one percent. Redundancy of words comes second followed by absence of words, then with mistaken use of parts of speech following them. In addition, much less frequently though they occur, mistaken use of comparatives and superlatives, singular and plural forms and modal verbs are indispensable parts of lexical errors.

Furthermore, it is revealed in Table1 that syntactic errors also abound in proofreading and error correction. In the TEM-8 papers from 2012 to 2019, errors about tense and voice, attributive and nominal clauses and non-finite verbs are the top three frequently checked.

Lastly, textual errors are contained to primarily test L2 learners' language proficiency about cohesion, which individually takes up about 21 percent of all errors, only after mistaken use of words. Cohesion, requiring a comprehensive knowledge of language learners, is the most difficult to handle.

However, a limited number of TEM- 8 papers are employed to sort out language forms included in proofreading and error correction. Therefore, a limited number of language forms are correspondingly categorized on the table, even though most of them are covered. 
Table 1 Classification of Language Forms in Proofreading and Error Correction

\begin{tabular}{|c|c|c|c|c|}
\hline \multicolumn{2}{|c|}{ Category } & Sub-category & Number & Frequency \\
\hline \multirow{8}{*}{1} & \multirow{7}{*}{ Lexical errors } & Redundancy of words & 7 & 0.0875 \\
\hline & & Absence of words & 6 & 0.075 \\
\hline & & Mistaken use of words & 21 & 0.265 \\
\hline & & Mistaken use of parts of speech & 4 & 0.05 \\
\hline & & Mistaken use of comparatives and superlatives & 1 & 0.0125 \\
\hline & & Mistaken use of modals & 0 & 0 \\
\hline & & Mistaken use in singular and plural forms of nouns & 1 & 0.0125 \\
\hline & & Total & 40 & 0.5 \\
\hline \multirow{8}{*}{2} & \multirow{7}{*}{ Syntactic errors } & Tense and voice & 8 & 0.1 \\
\hline & & Concord & 0 & 0 \\
\hline & & Attributive and nominal clauses & 7 & 0.0875 \\
\hline & & Non-finite verbs & 7 & 0.0875 \\
\hline & & It-sentence pattern & 1 & 0.0125 \\
\hline & & Subjunctive mood & 0 & 0 \\
\hline & & Inversion & 0 & 0 \\
\hline & & Total & 23 & 0.2875 \\
\hline \multirow[t]{2}{*}{3} & Textual errors & Cohesion & 17 & 0.2125 \\
\hline & & Total & 17 & 0.2125 \\
\hline
\end{tabular}

\section{ANALYSIS OF ERRORS AND DISCUSSION}

Zhang concludes in her study that learners in China are prone to be influenced by Chinese, owing to cross-linguistic differences [6]. This influence occurs in all aspects of language structure. If both languages have the same forms or rules, L2 learners benefit from L1 during the process of acquisition, which is termed positive transfer. If L1 features are different from those of the target language, and learners easily make mistakes, then negative transfer is inferred [1]. Taking this kind of transfer into consideration, it is certain that the question type of proofreading and error correction is favored by so many examinations, let alone TEM-8. As a consequence, this paper further analyzes errors from the perspective of EA considerately, with the framework of Richards' error types, to enable test-takers progress in proofreading and error correction consciously.

\subsection{Overgeneralization}

Overgeneralization, one error type of Richards, indicates that a learner creates deviant structures on the basis of his experience of other or similar structures in the target language [5]. From the very beginning of learning English, what teachers hammer is that the definite article "the" must be added before unique nouns in English, such as the words "time" and "nature", because the definite article does not exist in Chinese. Therefore, good English learners have paid close attention to this rule. Conversely, they are apt to misuse the word "the".

In the proofreading and error correction of TEM-8 papers, use of "the" is tested several times.

Example1: One reason why we take the language for granted is that it usually happens so effortlessly, and most of time, so accurately. (TEM-8, 2013) (3)

The word "time" is unique, so "the" should be used before it.

Example2: ... when the study of cultural

Anthropology suggested that the linguistic barriers

Were insuperable and that the language (6)

Was entirely the product of culture, the view translation was impossible gained some currency, and with it that, if was attempted at all, it must be as

Literal as possible. (TEM-8, 2012)

The word "language" is regarded as a unique noun for many English majors. Here the use of the finite article "the" is probably overgeneralized. Apparently, overgeneralized use of "the" is common among learners.

\subsection{Ignorance of Restrictions}

Ignorance of restrictions means that learners fail to observe rules of existing structures [5]. Acquiring a different language from mother tongue, learners frequently ignore some rules or restrictions of the target language. Consequently, errors derived from ignorance of restrictions are bountiful.

Example3: "Plush" was clearly intended as a complimentary, a positive evaluation; that (3)

Mush I could tell it from the tone of voice and the context. (TEM-8. 2015)

In Example3, the word "complimentary" is an adjective, which cannot be used after the indefinite article "a" alone, for that "a" should be followed by nouns. L2 learners sometimes find it difficult to detect this kind of error, resulting from their ignorance of restrictions. The same error type occurs in the following example.

Example4: The final source of difficulty

Is that $C G$ results from non-standard

Ways of thinking language

And linguistic investigation. (TEM-8, 2019) 
In most cases, an objective clause should be after the word "think", or a preposition should be added between "think" and the following nouns or pronouns. But in the example above, nouns go directly after "think" without inserting "about".

\subsection{Incomplete Application of Rules}

Regarding the type "incomplete application of rules", learners acquire and maybe know rules, but they are unable to apply them completely or appropriately [5].

Example5: The only constant in life is change; the more one accepts one's strengths

And works towards dealing with their shortcomings, specially in the area of

communication skills, the better will be their interactions and the more their social popularity. (TEM-8, 2017)

Here it is clear that an adverb, not an adjective, should be in front of the phrase "in the area of". But learners sometimes transfer the adjective "special" incompletely into a false adverb form "specially".

\subsection{False Concepts Hypothesized}

As the term "false concepts" suggests, learners falsely comprehend distinctions between two words in the target language [5].

Example6: As we shall see, all these examples of what might be called language in exceptional circumstances reveal a great deal about the processes evolved in speaking. (TEM-8, 2013)(9)

The verb "evolve" shares "-volve" with another verb "involve", thus leading to a confusion of these two words" meaning among L2 learners. Actually, this always happens in the acquisition of both L1 and L2.

Example7: That is, the spotlight is on what mental capabilities are involving, what psychological factors play a role in the learning or acquisition, and whether the target language is learned in the classroom or acquired through social touch with native speakers. (TEM-8, 2014) (10)

In Example7, words "touch" and "interaction" are synonyms. But if one thing touches with another, their surfaces come into contact with each other, while if people interact, they communicate as they work or spend time together. Apparently, it is the communication of humans that is emphasized here. So, the word "touch" is inappropriate. Errors deriving from false concepts are frequently made in synonyms.

Analyzing errors with the help of Richards' error types renders L2 learners a clearer and more comprehensive understanding of proofreading and error correction in TEM-8 papers. However, it is difficult for learners to define the error type accurately because the four error types intermix with each other without a clear dividing line.

\section{DEFECTS AND SUGGESTIONS}

Taking a thorough view of these eight TEM-8 papers, identification of differences between words with similar meanings or spellings and cohesion are attached increasingly greater importance to.

After conducting such a meticulous analysis, it is clear that these eight TEM-8 papers are not always perfect. Firstly, several same language forms are tested repeatedly, such as the usage of "the" in TEM- 8 paper of 2013 and the adverb form of "special". Secondly, language forms concerning concord are not involved at all, and adequate stress is not put on such language forms as tense and voice and inversion, which are both significant in the process of learning English. Thirdly, some passages given in proofreading and error correction have similar topics, such as the topic "language" in TEM-8 papers of 2013 and 2014 and "communication" in TEM-8 papers of 2016 and 2017. Accordingly, it is suggested that more various language forms should be tested in proofreading and error correction and that the questions in it should be better designed.

\section{CONCLUSION}

This study has made a detailed classification of language forms included in proofreading and error correction, and profoundly analyzed errors employing Richard's error types. Conclusions are: 1) Language forms included in proofreading and error correction can be categorized into lexical, syntactic and textual errors, each with some sub-categories. 2) L2 learners are able to consciously analyze errors in proofreading and error correction from the perspective of EA with the framework of Richards' error type, to improve their own skills of dealing with proofreading and error correction.

But there are still deficiencies owing to a limited number of TEM-8 papers, along with the indistinct error categorization. Endeavoring to dig deeper into to what extent L2 learners can benefit from analysis of errors, more accurate data, more thorough experiments and more scientific research are advised to replenish in the future study.

\section{ACKNOWLEDGMENT}

This research was supported by "the Fundamental Research Funds for the Central Universities (WUT: 2020VI035)".

\section{REFERENCES}

[1] L. Xiao, "The effect of Self-explanation on students' ability in answering proofreading and error correction questions in TEM-8” [D], Hunan University, 2014.

[2] M. Saville-Troike, Introducing Second Language Acquisition, 2nd ed., 2011, Cambridge: Cambridge University Press. 
[3] M. Hou, "Contrastive Analysis, Error Analysis, and Interlanguage theory in second language acquisition research", Journal of Jishou University (Social Sciences Edition), vol.32, 2011, pp. 159-162. DOI:

10.13438/j.cnki.jdxb.2011.03.006

[4] W. Zhang and S. Yang, "An analysis of V-N collocation errors in CLEC", Journal of PLA University of Foreign Languages, vol.32, 2009, pp. 39-44.

[5] K. Johnson, An Introduction to Foreign Language Learning and Teaching, 1st ed., 2002, Beijing: Foreign Language Teaching and Research Press.
[6] J. Peng, “An addition to error classification in Error Analysis based on Chomsky's Universal Grammar from the cognitive perspective", Foreign Language and Literature, vol. 29, 2013, pp. 95-97.

[7] H. Zhang, "A revelation of the Error - Analysis theory to English writing teaching for postgraduates of non-English majors", Theory and Practice of Education, vol. 32, 2012, pp.48-50.

[8] W. Dai and Z. He, A New Concise Course in Linguistics for Students of English, 2nd ed., 2013, Shanghai: Shanghai Foreign Language Education Press. 